RESEARCH REPORT

\title{
Skin cancer in people with multiple sclerosis: a record linkage study
}

\author{
M J Goldacre, V Seagroatt, D Yeates, E D Acheson
}

J Epidemiol Community Health 2004;58:142-144

See end of article for authors' affiliations ....................

Correspondence to: $\operatorname{Dr} M J$ Goldacre, Unit of Health-Care Epidemiology, Department of Public Health, Institute of Health Sciences, Oxford University, Old Road, Oxford OX3 7LF, UK; michael.goldacre@ dphpc.ox.ac.uk

Accepted for publication 27 July 2003
Objective: The prevalence of multiple sclerosis (MS) varies with latitude: it increases with distance from the equator in both hemispheres. To seek evidence on whether solar radiation is a protective factor for MS, this study investigated whether skin cancer, as an indicator of solar radiation, is less common in people with MS than in others.

Design: Analysis of a database of linked hospital records and death certificates.

Setting: The Oxford Region of the National Health Service, England.

Subjects: A cohort comprising all people in the database with MS, and comparison cohorts of people with other diseases.

Results: Skin cancer was significantly less common in people with MS than in the main comparison cohort (rate ratio $0.49 ; 95 \%$ confidence interval 0.24 to 0.91 ). There was no general deficit of cancer in the MS cohort, and no deficit of skin cancer in cohorts of people with other autoimmune or neurological diseases. Conclusion: The findings support the hypothesis that solar radiation may have a protective influence on the development of MS.
$\mathrm{T}$ he aetiology of multiple sclerosis (MS) remains obscure, but its remarkable global distribution may provide a clue to part of its causation. Among Europeans who have settled in North America, South Africa, Australia, and New Zealand there is a close relation between the prevalence of the disease and distance from the equator. ${ }^{12}$ In North America MS is more common in Canada and the northern states of the USA than in the southern states, while in the southern hemisphere there is a regular gradation in the prevalence of MS in the opposite direction-from south to north. ${ }^{2}$ These findings led to the suggestion that "the more sunshine there is in a climate the less MS there appears to be" and the hypothesis that "such an influence could conceivably act directly, a certain skin dose of sunshine per unit time protecting the individual in some way". ${ }^{2}$ One of the sun associated factors that showed a strong inverse correlation with MS in North American studies was lack of winter sunshine. ${ }^{13}$ This suggests that a minimum exposure throughout the year may be necessary to confer protection. Recent work has suggested that, as a mechanism, solar radiation might be one of the factors that influence the development of MS through an effect on the immune system. ${ }^{3}$

It is generally accepted that the direct effect of solar radiation on skin is an important cause of skin cancer in fair skinned people. Most squamous and basal cell carcinomas in fair skinned people occur on sun exposed skin and are related to affected individuals' cumulative dose of solar radiation. Solar keratoses commonly precede the development of squamous cell carcinoma. There is also strong evidence that solar radiation contributes to the aetiology of melanoma of the skin in some way, but the association with duration of exposure to sunlight is less linear than that for the epithelial skin cancers. The association with melanoma may be related, in part, to intermittent, recreational sun exposure.

If solar radiation protects against MS, people with MS might have a lower than average risk of skin cancer. We tested this hypothesis using data from the Oxford record linkage study (ORLS). ${ }^{4}$

\section{METHOD}

The ORLS includes brief statistical abstracts of records of all hospital admissions (including day cases) in National Health Service (NHS) hospitals, and all deaths regardless of where they occurred, in defined populations within the former Oxford NHS region, from 1 January 1963 to 31 March 1999. The hospital data were collected routinely in the NHS as hospital discharge statistics. They exclude patients treated in the private sector. The data about deaths were obtained by the ORLS from death certificates. Data collection covered two health districts from 1963 (population 850 000), six districts from 1975 (population 1.9 million) and all the eight districts of the region from 1987 (population 2.5 million). The data for each person were linked together routinely as part of the Oxford region's health information systems.

The MS cohort was obtained by selecting records of people aged 15 years and over with an admission for MS. The admission date was that of the first recorded admission for MS. It is not necessarily the first ever admission, which could have occurred outside the area covered by the database or before the start of the database. A reference cohort was constructed by similarly selecting records of people aged 15 years and over who had been admitted for various medical and surgical conditions. This was drawn from a standard "reference" group of patients that has been used in other studies of inter-relations between diseases. ${ }^{4}$ We identified any subsequent inpatient or day case care for skin cancers, and for all cancers, in these cohorts. We considered that the cancer rates in the reference cohort would approximate to those in the general population of the region while permitting for migration from it (data on migration of people were not available). The International Classification of Diseases (ICD) codes in ICD7, ICD8, ICD9, and ICD10 used for MS were, respectively, 345, 340, 340, and G35. The codes

Abbreviations: MS, multiple sclerosis; ORLS, Oxford record linkage study 


\section{Key points}

- The aetiology of multiple sclerosis is unknown, but its prevalence increases with distance from the equator in both hemispheres.

- Exposure to solar radiation is one possible factor that might influence its development.

- We investigated whether skin cancer, as a marker of individual exposure to solar radiation, was less common in people with multiple sclerosis than others.

- It was less common.

- Our finding adds to ecological evidence that solar radiation may have a protective influence on the development of multiple sclerosis.

used for skin cancer, respectively, were 190-1, 172-3, 172-3, and C43-4.

The people in the comparison cohort were matched with those in the MS cohort on age (within five years), sex, district of residence, and calendar year of first admission. We calculated rates based on person years at risk, accumulating person years in the strata defined by age, sex, district, and calendar year. We took date of entry into each cohort as date of first admission for MS or comparison condition, and date of exit as date of subsequent admission for skin cancer, death, or 31 March 1999, whichever was the earliest. For each age-sex-district-year stratum the ratios of cancer rates in the MS cohort were calculated relative to those in the reference cohort. These ratios were then summed across strata to give an overall rate ratio. Its confidence interval, and $\chi^{2}$ statistics for its significance, were calculated as described. ${ }^{5}$ We divided the cases of skin cancer into two groups: malignant melanoma (ICD10 code C43 and its equivalents in earlier revisions of the ICD) and other skin cancers (C44 and equivalents). We calculated rate ratios for each type of skin cancer. In this calculation, for the few patients with records of both types we selected the cancer that was recorded first. The significance of the difference between the rate ratios for each type of skin cancer was assessed by a $\chi^{2}$ test of heterogeneity.

To test the specificity of the occurrence of skin cancer in people with MS, we used the same analytical procedure to study other cancers in the MS cohort. To test the specificity of MS, as a chronic disease, and any deficit of skin cancer, we also calculated the rate ratios for skin cancer in people with other chronic diseases with an autoimmune component, or with other neurological diseases.

\section{Implication}

- The association between latitude and multiple sclerosis may be explained, at least in part, by exposure to solar radiation.

\section{RESULTS}

Table 1 shows the age distribution of the patients with MS. There were 5004 patients in the MS cohort and over 430000 in the reference cohort (table 2). The mean age at first admission and average length of follow up for the MS cohort were similar to those for the other cohorts. Ten cases of skin cancer were recorded among the MS patients giving it a rate ratio of 0.49 , significantly lower than one (table 2). Restricting the analysis to malignant melanoma gave four cases and a non-significant rate ratio of 0.91 (95\% confidence interval: 0.25 to $2.34, p=0.97)$. Restricting to other skin cancer gave six cases and a significant rate ratio of $0.38(0.14$ to $0.82 ; \mathrm{p}=0.02)$. Although the two rate ratios appear to differ, they were based on small numbers and had wide confidence intervals. The $\chi^{2}$ test for heterogeneity showed that the difference between them could be attributed to chance $\left(\chi^{2}=1.9, \mathrm{df}=1, \mathrm{p}=0.16\right)$.

Cancer rates overall were not low in the MS cohort: in fact, they were slightly raised with a rate ratio of 1.15 ( 1.01 to 1.31: $\mathrm{p}=0.03$; based on 243 cancers in the MS cohort). The two other groups, those comprising other autoimmune or neurological disorders, had rate ratios for skin cancer that were close to one (table 2).

\section{DISCUSSION}

Our findings are based on observational data and so there is potential for bias and confounding. Potential sources of bias include the following. Firstly, the data only include people who were admitted to hospital as inpatients or day cases. Secondly, the group of patients with MS, and each group with the other conditions studied, includes prevalent as well as incident cases and may therefore selectively include those with long survival times. Thirdly, there could be potential bias in referral patterns for cancer in people with MS: for example, there might be increased medical surveillance, and a higher rate of detection of and/or referral for cancer, for people with MS than others. Fourthly, it is possible that people who develop a chronic condition such as MS might subsequently spend less, or more, time in the sun than others. These possibilities of bias were part of our reasoning for considering all cancers and not just skin cancer in people with MS, and for studying skin cancer in people with other

Table 1 Age distribution of people in the cohort with multiple sclerosis at first recorded admission: number and percentage of people

\begin{tabular}{lll}
\hline Age $(\mathbf{y})$ & Number & Percentage \\
\hline$<25$ & 295 & 5.9 \\
$25-29$ & 443 & 8.9 \\
$30-34$ & 534 & 10.7 \\
$35-39$ & 586 & 11.7 \\
$40-44$ & 552 & 11.0 \\
$45-49$ & 601 & 12.0 \\
$50-54$ & 558 & 11.2 \\
$55-59$ & 431 & 8.6 \\
$60-64$ & 371 & 7.4 \\
$>65$ & 623 & 12.4 \\
Unrecorded & 10 & 0.2 \\
Total & 5004 & 100.0 \\
\hline
\end{tabular}


Table 2 Number of patients in each cohort, number with skin cancer, and adjusted ratio of the rate of skin cancer in each cohort relative to that in the reference cohort (with $95 \%$ confidence intervals on the rate ratio)

\begin{tabular}{|c|c|c|c|c|c|c|c|c|}
\hline Cohort (ICD code) & $\begin{array}{l}\text { Number of } \\
\text { people }\end{array}$ & $\begin{array}{l}\text { Average } \\
\text { age }(y)\end{array}$ & $\begin{array}{l}\text { Average } \\
\text { follow up (y) }\end{array}$ & $\begin{array}{l}\text { Total person } \\
\text { years }\end{array}$ & $\begin{array}{l}\text { Number with } \\
\text { skin cancer }\end{array}$ & $\begin{array}{l}\text { Adjusted rate } \\
\text { ratio* }\end{array}$ & $\begin{array}{l}95 \% \\
\text { Confidence } \\
\text { interval }\end{array}$ & p Value \\
\hline \multicolumn{9}{|l|}{ Main comparison } \\
\hline Reference cohort† & 432091 & 45 & 11.3 & 4876672 & 2284 & 1 & - & - \\
\hline Multiple sclerosis $\ddagger$ & 5004 & 46 & 10.2 & 51032 & 10 & 0.49 & 0.24 to 0.91 & 0.03 \\
\hline \multicolumn{9}{|l|}{ Other comparisons } \\
\hline Autoimmune conditions§ & 39831 & 46 & 10.4 & 414932 & 193 & 1.06 & 0.91 to 1.24 & 0.45 \\
\hline Diseases of central nervous system & 27274 & 50 & 8.1 & 219863 & 125 & 1.01 & 0.83 to 1.22 & 0.95 \\
\hline
\end{tabular}

*Adjusted for age in five year bands, sex, district of residence and calendar year. tInternational Classification of Diseases codes, ninth revision: acute respiratory infections (460-6), dental treatment (520-1), deflected nasal septum, nasal polyp 470, 471), inguinal hernia (550), diseases of nail (703), sebaceous cyst (706.2), internal derangement of knee (717), bunion (727.1), selected fractures, dislocations, sprains and strains $(810-6,823-6,830-9,840-8)$, superficial injury and contusion (910-24), otitis externa, otitis media (380-2), haemorrhoids (455); and Office of Population Census and Surveys operation codes, fourth revision: appendicectomy (H01-3) tonsillectomy, adenoidectomy (E20, F24, F36), hip, knee arthroplasty (W37-42). †See text for ICD codes. §Diabetes (250), rheumatoid arthritis (714), inflammatory bowel disease (555-6); restricted to those aged 15-64 at admission because of large numbers of elderly people with these diseases. - Stroke (430-8), motor neurone disease (335), other neurological diseases (330, 333-4, 341, 344-348); restricted to those aged 15-64 at admission because of large numbers of elderly people with these diseases.

chronic conditions. The facts that the deficit was specific to skin cancer and MS, and that the study was undertaken to test the single a priori hypothesis that sun exposure might protect against MS, add strength to our finding. It is also noteworthy that the association between MS and nonmelanoma skin cancers was stronger than that between MS and melanoma, albeit not significantly different from it. The association between sun exposure and skin cancer is itself stronger for non-melanoma skin cancer than for melanoma.

The underlying pathology of MS is that in susceptible people an initiating event, often probably a virus infection, leads to an autoimmune process that destroys myelin tissue. ${ }^{67}$ It has been postulated that solar radiation, and in particular ultraviolet radiation, modulates the processes between the initiating event, the autoimmune response, and the development of MS. ${ }^{67}$ Possible mechanisms for the action of ultraviolet radiation include suppressor effects on the immune system, perhaps mediated via changes to vitamin $\mathrm{D}_{3}$ or melatonin production. McMichael and Hall ${ }^{6} 7$ and van der Mei et $a l^{8}$ have discussed possible mechanisms in detail.

In a recent ecological study of MS and climate data in Australia, geographical variation in MS prevalence was found to be closely associated with variation in regional levels of measured ultraviolet radiation. ${ }^{8}$ The correlation between age standardised MS prevalence and mean annual levels of ultraviolet radiation in different Australian locations was $-0.91(p<0.01)$. The Australian study also showed significant and strong inverse correlations between the geographical distribution of melanoma incidence and that of MS prevalence. In a recent study in the United States, residential and occupational exposure to sunlight was compared in two large samples of people who had died respectively from MS and non-melanoma skin cancer. ${ }^{9}$ As expected, mortality from skin cancer was positively associated with both categories of exposure to sunlight: the odds ratio for the combined effect of residential and occupational exposure in the highest compared with the lowest sunlight exposure category was 1.38 (95\% CI 1.12 to 1.69 ). By contrast, MS was negatively associated with sunlight exposure: the odds ratio for the combined effect in the highest sunlight exposure category was 0.24 (95\% CI 0.15 to 0.38 ).

As far as we know, ours is the first study to provide evidence about MS and a marker of solar radiation at an individual patient level as distinct from a population level.* In conclusion, our cohort study adds to the ecological and occupational evidence from previous studies that solar radiation influences the processes that underlie the development of MS.

\section{CONTRIBUTORS}

E D Acheson formulated the hypotheses about sunlight and MS and about skin cancer and MS. M J Goldacre planned and designed the study. Both have been directors of the Oxford record linkage study. V Seagroatt and D Yeates contributed to study design and analysed the data. M J Goldacre wrote the first draft and all contributed to the final manuscript.

\section{Authors' affiliations}

M J Goldacre, V Seagroatt, D Yeates, Unit of Health-Care

Epidemiology, Department of Public Health, Institute of Health Sciences, Oxford University, Oxford, UK

E D Acheson, Department of Epidemiology, Public Health, University College London, Medical School, London, UK

Funding: The Unit of Health-Care Epidemiology is currently funded for analyses of the Oxford Record Linkage Study by the Research and Development Directorate of the Department of Health and Social Care (South).

Conflicts of interest: none declared.

\section{REFERENCES}

1 Acheson ED, Bachrach CA, Wright FM. Some comments on the relationship of the distribution of multiple sclerosis to latitude, solar radiation, and other variables. Acta Psychiat Neurol Scand 1960;35(suppl 147):132-47.

2 Acheson E, ed. Multiple sclerosis in British Commonwealth countries in the Southern Hemisphere. Brit J Prev Soc Med 1961;15:118-25.

3 Beebe GW, Kurtzke JF, Kurland LT, et al. Studies on the natural history of multiple sclerosis. 3: epidemiologic analysis of the Army experience in world war 2 Neurology 1967;17:1-17.

4 Goldacre MJ, Kurina L, Yeates D, et al. Use of large medical databases to study associations between diseases. Q J Med 2000;93:669-75.

5 Breslow NE, Day NE. Statistical methods in cancer research, volume II. The design and analysis of cohort studies. IARC scientific publication no 82. Lyon: International Agency for Research in Cancer, 1987:103-15.

6 McMichael AJ, Hall AJ. Does immunosuppressive ultraviolet radiation explain the latitude gradient for multiple sclerosis? Epidemiology 1997;8:642-5.

7 McMichael AJ, Hall AJ. Multiple sclerosis and ultraviolet radiation: time to shed more light. Neuroepidemiology 2001;20:165-7.

8 van der Mei IAF, Ponsonby AL, Blizzard L, et al. Regional variation in multiple sclerosis prevalence in Australia and its association with ambient ultraviolet radiation. Neuroepidemiology $2001 ; 20$ : 168-74.

9 Freedman DM, Dosemeci M, Alavanja MCR. Mortality from multiple sclerosis and exposure to residential and occupational solar radiation: a case-control study based on death certificates. Occup Environ Med 2000;57:418-21.

10 Van der Mei IAF, Ponsonby AL, Dwyer J. Past exposure to sun, skin phenotype, and risk of multiple sclerosis: case-control study. BMJ 2003;327:316-20 